vanhee celine (Orcid ID: 0000-0002-6634-3706)

\title{
Substandard and falsified antimicrobials: A potential biohazard in disguise?
}

Yaxin Tie ${ }^{1,2}$, Erwin Adams ${ }^{2, \#}$, Eric Deconinck ${ }^{1, \#}$, Celine Vanhee ${ }^{1, \#, *}$

1 Scientific Direction Chemical and Physical Health Risks, Section Medicines and Health Products, Sciensano, J. Wytsmansstraat 14, 1050 Brussels, Belgium

2 KU Leuven, University of Leuven, Department of Pharmaceutical and Pharmacological Sciences, Pharmaceutical Analysis, Herestraat 49, O\&N2, PB 923, B-3000 Leuven, Belgium

* Corresponding author

\# Equally contributing project leaders

This article has been accepted for publication and undergone full peer review but has not been through the copyediting, typesetting, pagination and proofreading process which may lead to differences between this version and the Version of Record. Please cite this article as doi: $10.1002 /$ dta. 2740 


\section{Introduction}

It is widely acknowledged that the threat of counterfeited and falsified medicines to public health remains a global issue [1]. In order to overcome country, region or organizational differences in semantics and to simplify the terminology, the World Health Organization (WHO) has adopted, since 2017, the term "substandard and falsified (SF) medical products" to describe substandard medical products, unregistered/unlicensed medical products and falsified medical products [2]. SF versions can be found for almost all different kinds of medicinal products, including lifesaving drugs such as antimalarial medication, antimicrobials, antivirals and insulin. Among these lifesaving drugs, antimicrobials are one of the most popular subjects of these malignant activities [2].

The last decade has been marked with numerous actions that were taken to prevent SF medical products from infiltrating legal supply chains [3]. In Europe specifically, the European Directive (2011/62/EU) on Falsified Medicines for human use has been implemented in February 2019. This directive provides a preventive system for falsified medicines, e.g. safety features (unique identifier and tamper-evident packaging) are obligatory for medical products [4]. Additionally to the regulatory efforts, also many efficient analytical methods were developed for the analysis and risk evaluation of SF medical products [5-10]. The majority of these analytical findings is focused on the chemical characterization, i.e. presence of the active pharmaceutical ingredients (APIs) and impurities. However, also microbiological contamination of these SF medical products could have an impact on the risks these products represent. Indeed, it has been reported that clindamycin injectables, produced in China, led to nine fatalities in 2006. The cause of this lethal outcome was due to the presence of bacteria that were able to proliferate as a result of an inadequate sterilization procedure [11]. Moreover, in 2016, 21 patients were injected with counterfeit bevacizumab in 2 Iranian hospitals. These intravitreal injections resulted in an outbreak of fulminant endophthalmitis that was very likely caused by the presence of virulent strains of Escherichia coli and Citrobacter sp. in the falsified product [12]. Additionally, Janvier et al. found that two injectable illegal polypeptide drugs, purchased from an illicit online peptide provider disguised as a research company, were contaminated with a haemolytic strain of Bacillus cereus [13]. Although the above examples described the potential hazard of SF injectable drugs, also microbial contamination of oral preparations with pathological bacteria 
that can survive the acidic environment of the stomach, have been described for the popular illegal SF phosphodiesterase-type 5 inhibitors [8].

Taking into account the popularity of antimicrobial drugs and the fact that they are utilized by vulnerable patients, the aim of this study was to investigate the microbial quality of 20 different SF antimicrobials. A total of 17 SF antimicrobials, that have to be taken orally (tablets or capsules) and that were confiscated by the Belgian Federal Agency for Medicines and Health Products (FAMHP), were analyzed and six of them were largely exceeding the compliance limits put forward in the United States Pharmacopeia (USP) [14] and European Pharmacopoeia (Ph. Eur.) [15]. Moreover, also one out of three injectable samples was found to be contaminated with a pathogenic yeast, Penicillium crustosum, known for its ability to produce neurotoxins [16].

2 Material and methods

\subsection{Experimental materials}

Thioglycollate broth with resazurin (THIO) and Trypcase soy broth (TSB) were bought from BioMérieux (Marcy-I'Etoile, France). Sabouraud chloramphenicol gentamicin agar (SCG) especially used for fungal organisms, Trypso-Casein-Soy agar (TCS), MacConkey agar with crystal violet (MCV) and Columbia Agar + 5\% Sheep Blood were all purchased from BioRad (Hercules, CA, USA). Sterile phosphate buffer saline (PBS) was purchased from ThermoFisher Scientific (Waltham, MA, USA) while sterile buffered saline peptone water with neutralizers $(=$ Buffered Sodium Chloride Peptone Solution with 0.3\% (w/v) Lecithin, 3\% (w/v) Tween and 0.1\% (w/v) Histidine) was bought from Merck (Darmstadt, Germany).

Bacterial reference strains of Pseudomonas aeruginosa (NCTC 10662), Staphylococcus aureus (ATCC 25923), Bacillus subtilis (ATCC 6633), Clostridium sporogenes (LMG 14743), Bacteroides fragilis (LMG 10263) and fungal strains of Candida albicans (IHEM 3731) and Aspergillus niger (IHEM 3766) were used as positive controls to ensure proper growth conditions. Sterile analytical filter units $0.45 \mu \mathrm{m}$ Nalgene ${ }^{\mathrm{TM}}$, used for the membrane filtration during the sterility test, were purchased from Thermo-Fisher Scientific (Rochester, NY, USA).

\subsection{Sample set}

Twenty illegal SF antibiotic samples were collected by inspectors from FAMHP. Three injectable samples including ampicillin, ceftriaxone and benzathine benzylpenicillin were subjected to sterility testing while the 17 other illegal SF antimicrobials (tablets or capsules) 
including amoxicillin, azithromycin, roxithromycin, metronidazole, griseofulvin, ofloxacin with ornidazole, doxycycline, tetracycline, erythromycin and norfloxacin were subject to bioburden testing. Additionally, three genuine amoxicillin samples purchased from a local pharmacy, were also analyzed for bioburden testing. The tested injectable samples were well sealed and all tablets and capsules involved in the study were preserved in blisters and did not pass their expiry date.

\subsection{Sterility testing}

The sterility testing was performed according to the International Pharmacopoeia 3.2 test for sterility [17], which has undergone pharmacopoeial harmonization of the Japanese Pharmacopoeia (JP), USP and Ph. Eur. Negative controls were performed on culture media, membrane filters, peptone with neutralisers, PBS buffer, sterility of the flow hood and the incubators. The broths of THIO and TSB were selected as growth media. Strains of Bacillus subtilis, Staphylococcus aureus, Escherichia coli, Pseudomonas aeruginosa, Clostridium sporogenes, Bacteroides fragilis, Candida albicans and Aspergillus niger were inoculated as positive controls. Aforementioned growth media and growth conditions fulfilled the growth promotion test of aerobes, anaerobes and fungi.

For sterility testing, five vials of each sample were randomly selected and subjected to both membrane filtration and direct inoculation of the culture medium. The outside surfaces of the sample vials were disinfected before they were put under the laminar flow hood for sterility testing. The vial content of ampicillin and ceftriaxone was dissolved in $5 \mathrm{~mL}$ peptone with neutralizers in order to obtain a concentration of $200 \mathrm{mg} / \mathrm{mL}$. The vial content of benzathine benzylpenicillin was dissolved in $8 \mathrm{~mL}$ peptone with neutralizers, resulting in a concentration of $225 \mathrm{mg} / \mathrm{mL}$. $1 \mathrm{~mL}$ of sample solution was either directly inoculated into the culture medium (TSB or THIO) or was applied to the membrane prior to membrane filtration and subsequent transfer of the filter to THIO or TSB medium. Each membrane filter with sample was washed 3 times with $100 \mathrm{~mL}$ PBS. The THIO broths were incubated at $32{ }^{\circ} \mathrm{C}$, while the broths with TSB were incubated at $25{ }^{\circ} \mathrm{C}$.

\subsection{Bioburden testing}

Bioburden testing aims to count aerobic microorganisms that are possibly present in pharmaceutical preparations. These microorganisms are grown to visible colonies and it is assumed that a colony is formed from one colony forming unit (CFU). Bioburden testing was performed based on Ph. Eur. 2.6.12. Microbiological examination of non-sterile products 
(oral products) using the membrane filtration method [15]. Negative controls were performed on culture media, membrane filters, peptone water, PBS buffer and swabs were taken to demonstrate the cleanliness of the flow hood and the incubators.

A total of 20 samples (tablets and capsules), including 17 SF antimicrobials and three genuine antimicrobials, were subjected to bioburden testing for oral products. Due to the nature of the samples, i.e. antimicrobials, it was impossible to remove the biocidal activity for their target organism.

\subsection{Fungal and bacterial identification}

When fungi were detected in the samples, they were analyzed by a validated matrix-assisted laser desorption ionization-time of flight mass spectrometry (MALDI-TOF-MS) system, coupled to MALDI Biotyper 3 software (Bruker Daltonics, Bremen, Germany) containing both commercially available spectra and in-house generated spectra [18,19]. Identification of the encountered bacteria was performed according to the procedure utilized by Janvier et al. [13].

\section{Results and discussion}

\subsection{Sterility testing}

SF injectable antimicrobials confiscated by the controlling agency were sealed in the glass vials (see Fig. 1A). It stands to reason that injectable drugs should be sterile. However, one vial out of five of ceftriaxone displayed fungal growth after 10 days of incubation on the TSB medium. The suspected fungus was found in both the directly inoculated fraction and in the fraction that underwent membrane filtration (see Fig. 1B). No growth was observed for the other samples of ceftriaxone, the samples of the other antibiotics, all negative controls (work space before, after and during the operation, membrane and used solutions and media) remained negative after two weeks, illustrating the small likelihood of an introduced contamination. Next, the fungal species was further isolated on a classical SCG plate, which was subsequently used as starting point to identify the species by means of MALDI-TOFMS. Startlingly, the obtained identification data indicated that the microorganism found in the sample corresponded to Penicillium crustosum [16,20]. Penicillium crustosum is a mold that is able to survive (or its spores) in environments characterized by low water activity, including dried powders [21]. Upon injection of this contaminated sample, this fungus (or its spores) would enter the circulatory system that could curtail the growth of unwanted intruders. Therefore, we verified if this species could also grow on Colombia Agar blood 
plates at $37^{\circ} \mathrm{C}$. After 3 days of incubation, mold colonies could be found on the plates (See Fig. 1C). This example demonstrates the potential biohazardous dangers related to the use of illegal antimicrobial injectables, where the consequences of a blood infection with $P$. crustosum could be disastrous for the already vulnerable patient. It has been reported that a couple had severe generalised tremors after eating food contaminated with $P$. crustosum, demonstrating that this fungus or its spores can survive the acid environment in the stomach [22]. Another case also reported a tremorgenic syndrome in a man as a result of the consumption of a beer that was contaminated with this mold [23]. Moreover, the neurotoxin produced by $P$. crustosum can lead to death of cerebellar granule neurons in vivo [24] and it has been shown that larger doses could cause seizers, massive liver necrosis, convulsions and death in vivo [25]. Since the symptoms induced by this fungus are easily confused with other neurological diseases, the reports of infections caused by $P$. crustosum may be under-reported [26].

\subsection{Bioburden testing}

According to the Ph. Eur., the maximum acceptance criteria for the total aerobic microbial count (TAMC) is set at $200 \mathrm{CFU}$ per $\mathrm{mL}$ or gram of product, while the maximum acceptance criteria for the total yeast and mold count (TYMC) is set on $20 \mathrm{CFU}$ per mL or gram of product $(=20 \mathrm{CFU} / \mathrm{mL}$ ). Moreover, for these types of samples (oral administration), no E. coli are allowed to be present [15]. Our obtained data display that the tested genuine samples were compliant for both the TAMC and the TYMC limits (see Fig. 2A and Fig. 2B). Moreover, none of the analysed SF drugs exceeded the maximum acceptance limits for the TAMC, which was expected since the API's present in these samples belong to the class of antimicrobials. On the other hand, six out of the $17 \mathrm{SF}$ antimicrobials were not compliant for the presence of fungi (see Fig. 2B-C and Fig. 3). Unsettlingly, four samples surpassed the maximum acceptance limit by at least a factor ten (see Fig. 2C), indicating a severe fungal contamination.

All suspected isolates were then further identified by means of MALDI-TOF-MS coupled to the extensive in-house database of the BCCM/IHEM (Belgian co-ordinated collections of micro-organisms from the Mycology Laboratory of the Institute of Hygiene and Epidemiology), the Belgian co-ordinated fungi collection [27]. In total 17 fungi-like isolates were analyzed. The results showed that two isolates contained Penicillium chrysogenum, 14 isolates were identified as Penicillium rubens and one isolate was positive for Penicillium nalgiovense. All fungi are common in subtropical regions. P. chrysogenum is known to be 
able to act as pathogen and/or allergen, inducing serious intoxication and damage to humans [28]. Indeed, it has been reported that a 57-year-old woman suffered from necrotizing pneumonia caused by P. chrysogenum [29]. Moreover, also a case of necrotizing esophagitis occurring in a patient with a compromised immune system was attributed to this fungus [30]. Even a patient without immunological deficiencies was not exempted from central nervous system infection due to this mold [31]. Additionally, P. chrysogenum is widely known as an allergen and sensitive individuals may suffer from skin allergies and breathing problems [32]. $P$. rubens, on the other hand, has rarely been linked to human disease, but it was frequently encountered in clinical samples in the United States [28]. Interestingly, a recent study demonstrated that $P$. rubens could produce a mycotoxin, ochratoxin A, which can cause intoxication of the kidneys and the liver[33]. The occurrence of $P$. nalgiovense, an occupational and contact allergen, was also found in some samples, indicating the unqualified manufacturing process of SF antimicrobials [34].

In addition to the fungal contaminations, it is also worth mentioning that one azithromycin sample (sample SF9) although conform for the TAMC, did contain a bacterial species identified as a part of the Bacillus group. Fortunately, further investigation revealed the absence of pathogenic genes that are present in Bacillus species associated with food borne diseases.

Identification of suspected fungi or bacteria is not required by the bioburden testing as prescribed in the Ph. Eur. [15]. However, with this example we want to demonstrate that although these samples passed the TAMC and TYMC, it might also be important to identify the species present or assess their pathological potential. Moreover, another sample, SF2, was found to be compliant with the Ph. Eur. limit for TYMC (see Fig. 2C), but it was contaminated with $P$. rubens and $P$. chrysogenum (see Table 1), species known to be responsible for human mycoses [35-37]. These aforementioned findings clearly demonstrate the possible microbiological hazard that this popular class of SF medicines poses to the already vulnerable patients.

4 Conclusion

This study examined the microbiological quality of $20 \mathrm{SF}$ antimicrobials confiscated by the FAMHP. Three injectable samples were submitted to sterility tests while the tablets and capsules were tested for their bioburden.

For sterility testing, one vial, out of a total of 15 vials ( 5 vials per product), was contaminated with a pathogenic neurotoxin producing fungus. 
Moreover, six out of $17 \mathrm{SF}$ antimicrobials (tablets or capsules) were not compliant for bioburden testing of the TYMC prescribed by $\mathrm{Ph}$. Eur. [15] and four out of these six antimicrobials exceeded the maximum acceptance limit with a factor of ten and can be considered severely contaminated and even potentially dangerous. Indeed, it has to be admitted that unknown microorganisms found in SF samples even below the Ph. Eur. limits, could still possibly convey serious detrimental health effects to vulnerable patients. Since patients treated with antimicrobials already suffer from infections, SF antimicrobials contaminated with microorganisms could endanger the treatment procedure or even induce new infections.

\section{Acknowledgements}

The authors would like to acknowledge the kind colleagues from the BCCM/IHEM and the colleagues from the foodborne pathogens for the collaboration on the identification of the respectively found fungi and bacteria and to thank the China Scholarship Council (CSC, File No. 201607650019). Moreover, we would also like to mention that we are very grateful to the Federal Agency for Medicines and Health Products (FAMHP) for providing us with the illegal antimicrobial samples.

\section{Reference}

[1] World Health Organization. WHO global surveillance and monitoring system for substandard and falsified medical products; 2017. http://www.who.int/medicines/regulation/ssffc/publications/GSMS_Report.pdf?ua=1. Accessed February 4, 2019.

[2] World Health Organization. 1 in 10 medical products in developing countries is substandard or falsified; 2017. http://www.who.int/en/news-room/detail/28-11-2017-1in-10-medical-products-in-developing-countries-is-substandard-or-falsified. Accessed September 21, 2019.

[3] Venhuis BJ, Oostlander AE, Di Giorgio D, Mosimann R, Plessis I. Series Drug safety in oncology 3 Oncology drugs in the crosshairs of pharmaceutical crime. Lancet Oncol. 2018; 19(4): e209-e217. doi:10.1016/S1470-2045(18)30101-3.

[4] European Parliament, European Council. Directive 2011/62/EU. Off J Eur Union. 2011; L174: 74-87. 
[5] Vanhee C, Janvier S, Desmedt B, et al. Analysis of illegal peptide biopharmaceuticals frequently encountered by controlling agencies. Talanta. 2015; 142: 1-10. doi:10.1016/j.talanta.2015.04.022.

[6] Janvier S, De Sutter E, Wynendaele E, De Spiegeleer B, Vanhee C, Deconinck E. Analysis of illegal peptide drugs via HILIC-DAD-MS. Talanta. 2017; 174: 562-571. doi:10.1016/j.talanta.2017.06.034.

[7] Tie Y, Vanhee C, Deconinck E, Adams E. Development and validation of chromatographic methods for screening and subsequent quantification of suspected illegal antimicrobial drugs encountered on the Belgian market. Talanta. 2019; 194: 876-887. doi:10.1016/j.talanta.2018.10.078.

[8] Pullirsch D, Bellemare J, Hackl A, Trottier Y-L, Mayrhofer A, Schindl H, Taillon C, Gartner C, Hottowy B, Beck G, Gagnon J. Microbiological contamination in counterfeit and unapproved drugs. BMC Pharmacol. Toxicol. 2014; doi:10.1186/2050-6511-15-34.

[9] Johansson M, Fransson D, Rundlöf T, Huynh NH, Arvidsson T. A general analytical platform and strategy in search for illegal drugs. J Pharm Biomed Anal. 2014; 100: 215-229. doi:10.1016/j.jpba.2014.07.026.

[10] Cheng Q, Shou L, Chen C, Shi S, Zhou M. Application of ultra-high-performance liquid chromatography coupled with LTQ-Orbitrap mass spectrometry for identification, confirmation and quantitation of illegal adulterated weight-loss drugs in plant dietary supplements. $J$ Chromatogr B. 2017; 1064: 92-99. doi:10.1016/j.jchromb.2017.09.009.

[11] Jing Y, Kelton CML, Li X, Guo JJ. Lethal drug probe in China: the case of Xinfu clindamycin. Pharmacoepidemiol Drug Saf. 2007; 16(8): 928-932. doi: $10.1002 /$ pds. 1437

[12] Entezari M, Karimi S, Ahmadieh H, Mahmoudi AH, Parhizgar H, Yaseri M. A large outbreak of fulminant bacterial endophthalmitis after intravitreal injection of counterfeit bevacizumab. Graefes Arch Clin Exp Ophthalmol. 2016; 254(9): 18511856. doi:10.1007/s00417-016-3426-7.

[13] Janvier S, Wattijn E, Botteldoorn N, De Spiegeleer B, Deconinck E, Vanhee C. Are injectable illegal polypeptide drugs safe? Case report demonstrating the presence of 
haemolytic Bacillus cereus in 2 illegal peptide drugs. Drug Test Anal. 2018; 10(4): 791-795. doi:10.1002/dta.2304.

[14] United States Pharmacopeial Convention. United States Pharmacopeia and National Formulary (USP 41-NF 36). Rockville, MD, USA; 2018.

[15] European Pharmacopoeia, 9th ed. Strasbourg, France: Council of Europe; 2019.

[16] Altug T. Introduction to toxicology and food. 1st Ed. Boca Raton, USA, CRC Press; 2002. doi:https://doi.org/10.1201/b13578.

[17] World Health Organization. The International Pharmacopoeia, Eighth ed, Geneva; 2018. https://search.library.wisc.edu/catalog/9910066369602121.

[18] Normand AC, Cassagne C, Gautier M, et al. Decision criteria for MALDI-TOF MSbased identification of filamentous fungi using commercial and in-house reference databases. BMC Microbiol. 2017; 17(1): 17-25. doi:10.1186/s12866-017-0937-2.

[19] Normand AC, Becker P, Gabriel F, et al. Validation of a new web application for identification of fungi by use of matrix- assisted laser desorption ionization - time of flight mass spectrometry. $J$ Clin Microbiol. 2017; 55(9): 2661-2670. doi:10.1128/JCM.00263-17.

[20] Kalinina SA, Jagels A, Cramer B, Geisen R, Humpf HU. Influence of environmental factors on the production of penitrems A-F by Penicillium crustosum. Toxins. 2017; 9(7): 210-231. doi:10.3390/toxins9070210.

[21] Leggieri MC, Decontardi S, Bertuzzi T, Pietri A, Battilani P. Modeling growth and toxin production of toxigenic fungi signaled in cheese under different temperature and water activity regimes. Toxins. 2017; 9(1): 4-20. doi:10.3390/toxins9010004.

[22] Lewis PR, Donoghue MB, Hocking AD, Cook L, Granger LV. Tremor syndrome associated with a fungal toxin: sequelae of food contamination. Med J Aust. 2005; 182(11): 582-584. doi: 10.5694/j.1326-5377.2005.tb06819.x

[23] Cole RJ, Dorner JW, Cox RH, Raymond LW. Two classes of alkaloid mycotoxins produced by Penicillium crustosum thom isolated from contaminated beer. J Agric Food Chem. 1983; 31(3): 655-657. doi:10.1021/jf00117a045.

[24] Berntsen HF, Wigestrand MB, Bogen IL, Fonnum F, Walaas SI, Moldes-Anaya A. Mechanisms of penitrem-induced cerebellar granule neuron death in vitro: Possible 
involvement of $\mathrm{GABA}_{\mathrm{A}}$ receptors and oxidative processes. Neurotoxicology. 2013; 35: 129-136. doi:10.1016/j.neuro.2013.01.004.

[25] Breton P, Bizot JC, Buee J, De La Manche I. Brain neurotoxicity of Penitrem A: electrophysiological, behavioral and histopathological study. Toxicon. 1998; 36(4): 645-655. doi:10.1016/S0041-0101(97)00084-6.

[26] ScienceDaily. Fungus-induced neurological disease: An underestimated risk for animals and humans; 2011. https://www.sciencedaily.com/releases/2011/12/111215094811.htm Accessed September 16, 2019.

[27] Dupont D, Normand AC, Persat F, Hendrickx M, Piarroux R, Wallon M. Comparison of matrix- assisted laser desorption ionization time of flight mass spectrometry (MALDI-TOF MS) systems for the identification of moulds in the routine microbiology laboratory. Clin Microbiol Infect. 2019; 25(7): 892-897. doi:10.1016/J.CMI.2018.10.013.

[28] Guevara-Suarez M, Sutton DA, Cano-Lira JF, et al. Identification and antifungal susceptibility of penicillium-like fungi from clinical samples in the United States. $J$ Clin Microbiol. 2016; 54(8): 2155-2161. doi:10.1128/JCM.00960-16.

[29] D'Antonio D, Violante B, Farina C, Sacco R, Angelucci D, Masciulli M, Iacone A, Romano F. Necrotizing pneumonia caused by Penicillium chrysogenum. J Clin Microbiol. 1997; 35(12): 3335-3337.

[30] Hoffman M, Bash E, Berger SA, Burke M, Yust I. Fatal necrotizing esophagitis due to Penicillium chrysogenum in a patient with acquired immunodeficiency syndrome. Eur J Clin Microbiol Infect Dis. 1992; 11(12): 1158-1160. doi:10.1007/BF01961135.

[31] Kantarcıoğlu AS, Apaydın H, A. Yücel, De Hoog GS, Samson RA, Vural M, Özekmekçi S, Central nervous system infection due to Penicillium chrysogenum. Mycoses. 2004; 47(5): 242-248. doi:10.1111/j.1439-0507.2004.00974.x.

[32] Shen HD, Chou H, Tam MF, Chang CY, Lai HY, Wang SR. Molecular and immunological characterization of Pen ch 18, the vacuolar serine protease major allergen of Penicillium chrysogenum. Allergy. 2003; 58(10): 993-1002. doi:10.1034/j.1398-9995.2003.00107.x.

[33] Zhang X, Li Y, Wang H, Gu X, Zheng X, Wang Y, Diao J, Peng Y, Zhang H. 
Screening and identification of novel ochratoxin A-producing fungi from grapes. Toxins. 2016; 8(11): 1-14. doi:10.3390/toxins8110333.

[34] Brito FF, Mur P, Leal JA, et al. Penicillium nalgiovense as an occupational and contact allergen. J Allergy Clin Immunol. 2003; 112(1): 213-215. doi: 10.1067/mai.2003.1566.

[35] Lyratzopoulos G, Ellis M, Nerringer R, Denning DW. Invasive infection due to Penicillium species other than P. marneffei. J. Infect. 2002; 45(3): 184-195. doi:10.1016/S0163-4453(02)91056-X.

[36] López-Martínez R, Neumann L, González-Mendoza A. Case Report: cutaneous penicilliosis due to Penicillium chrysogenum. Mycoses. 1999; 42(4): 347-349.

[37] Barcus AL, Burdette SD, Herchline TE. Intestinal invasion and disseminated disease associated with Penicillium chrysogenum. Ann Clin Microbiol Antimicrob. 2005; 4(21): 1-4. doi:10.1186/1476-0711-4-21.

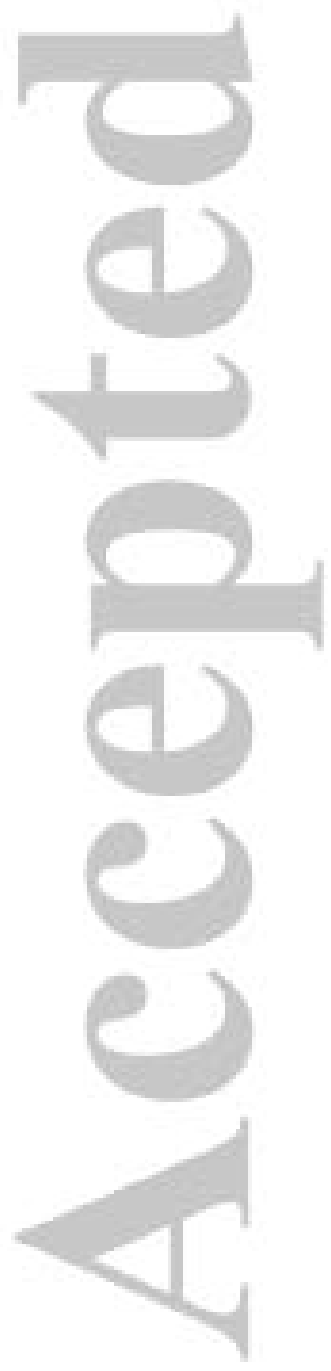


Table 1. Overview of the total aerobic microbial count and the total yeast and mold count revealed by the bioburden testing of the different SF antimicrobials and the subsequent identification of the different strains encountered in those samples.

\begin{tabular}{|c|c|c|c|c|c|c|c|}
\hline Drug name & Code & $\begin{array}{l}\text { TAMC } \\
\text { (CFU) }\end{array}$ & $\begin{array}{l}\text { Ph. Eur. } \\
\text { TAMC } \\
\text { (limit } \\
\text { CFU } \\
=200 \text { ) }\end{array}$ & $\begin{array}{l}\text { TYMC } \\
\text { (CFU) }\end{array}$ & $\begin{array}{l}\text { Ph. Eur. } \\
\text { TYMC } \\
\text { (limit } \\
\text { CFU=20) }\end{array}$ & $\begin{array}{l}\text { Identification } \\
\text { of isolates }\end{array}$ & $\begin{array}{l}\text { Activity } \\
\text { against }\end{array}$ \\
\hline \multirow{11}{*}{$\begin{array}{l}\text { Amoxicillin+clavulanic } \\
\text { acid }\end{array}$} & G1 & / & Conform & / & Conform & / & \multirow{11}{*}{$\begin{array}{c}\text { Most } \\
\text { gram- } \\
\text { positive } \\
\text { bacteria } \\
\text { and some } \\
\text { gram- } \\
\text { negative } \\
\text { bacteria }\end{array}$} \\
\hline & G2 & / & Conform & / & Conform & / & \\
\hline & G3 & / & Conform & / & Conform & / & \\
\hline & SF1 & / & Conform & / & Conform & / & \\
\hline & SF2 & / & Conform & 15 & Conform & $\begin{array}{l}\text { P. rubens, } P \text {. } \\
\text { chrysogenum }\end{array}$ & \\
\hline & SF3 & / & Conform & / & Conform & / & \\
\hline & SF4 & / & Conform & 15 & Conform & $\begin{array}{l}P \text {. rubens, } P \text {. } \\
\text { nalgiovense }\end{array}$ & \\
\hline & SF5 & / & Conform & 380 & $\begin{array}{c}\text { Not } \\
\text { conform }\end{array}$ & P. rubens & \\
\hline & SF6 & / & Conform & / & Conform & / & \\
\hline & SF7 & / & Conform & 15 & Conform & P. rubens & \\
\hline & SF8 & / & Conform & 275 & $\begin{array}{c}\text { Not } \\
\text { conform }\end{array}$ & P. rubens & \\
\hline Azithromycin & SF9 & 1 & Conform & 25 & $\begin{array}{c}\text { Not } \\
\text { conform }\end{array}$ & $\begin{array}{c}\text { P. rubens } \\
\text { Bacillus sp. } \\
\text { (TAMC) }\end{array}$ & $\begin{array}{c}\text { Gram- } \\
\text { positive } \\
\text { and } \\
\text { negative }\end{array}$ \\
\hline Roxithromycin & SF10 & / & Conform & / & Conform & / & $\begin{array}{c}\text { Gram- } \\
\text { negative }\end{array}$ \\
\hline Erythromycin & SF11 & / & Conform & 275 & $\begin{array}{c}\text { Not } \\
\text { conform }\end{array}$ & P. rubens & $\begin{array}{l}\text { Gram- } \\
\text { positive }\end{array}$ \\
\hline
\end{tabular}




\begin{tabular}{|c|c|c|c|c|c|c|c|}
\hline 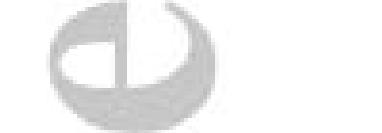 & & & & & & & $\begin{array}{c}\text { and } \\
\text { negative }\end{array}$ \\
\hline Doxycycline & SF12 & / & Conform & / & Conform & / & $\begin{array}{l}\text { Gram- } \\
\text { negative }\end{array}$ \\
\hline Tetracycline & SF13 & / & Conform & 580 & $\begin{array}{c}\text { Not } \\
\text { conform }\end{array}$ & P. rubens & $\begin{array}{l}\text { Gram- } \\
\text { negative }\end{array}$ \\
\hline Norfloxacin & SF14 & / & Conform & / & Conform & / & $\begin{array}{c}\text { Gram- } \\
\text { positive } \\
\text { and } \\
\text { negative }\end{array}$ \\
\hline Ofloxacin+ornidazole & SF15 & / & Conform & / & Conform & / & $\begin{array}{c}\text { Gram- } \\
\text { positive } \\
\text { and } \\
\text { negative }\end{array}$ \\
\hline Griseofulvin & SF16 & / & Conform & 25 & $\begin{array}{c}\text { Not } \\
\text { conform }\end{array}$ & P. rubens & $\begin{array}{c}\text { Antifungal } \\
\text { agent }\end{array}$ \\
\hline Metronidazole & SF17 & / & Conform & / & Conform & / & $\begin{array}{c}\text { Gram- } \\
\text { negative }\end{array}$ \\
\hline
\end{tabular}

TAMC: Total aerobic microbial count, TYMC: Total yeast and mold count, CFU: colony forming unit.

"Gx" indicates genuine samples and "SFx" indicates substandard and falsified samples.

"I" indicates that no colony was found in the sample. 


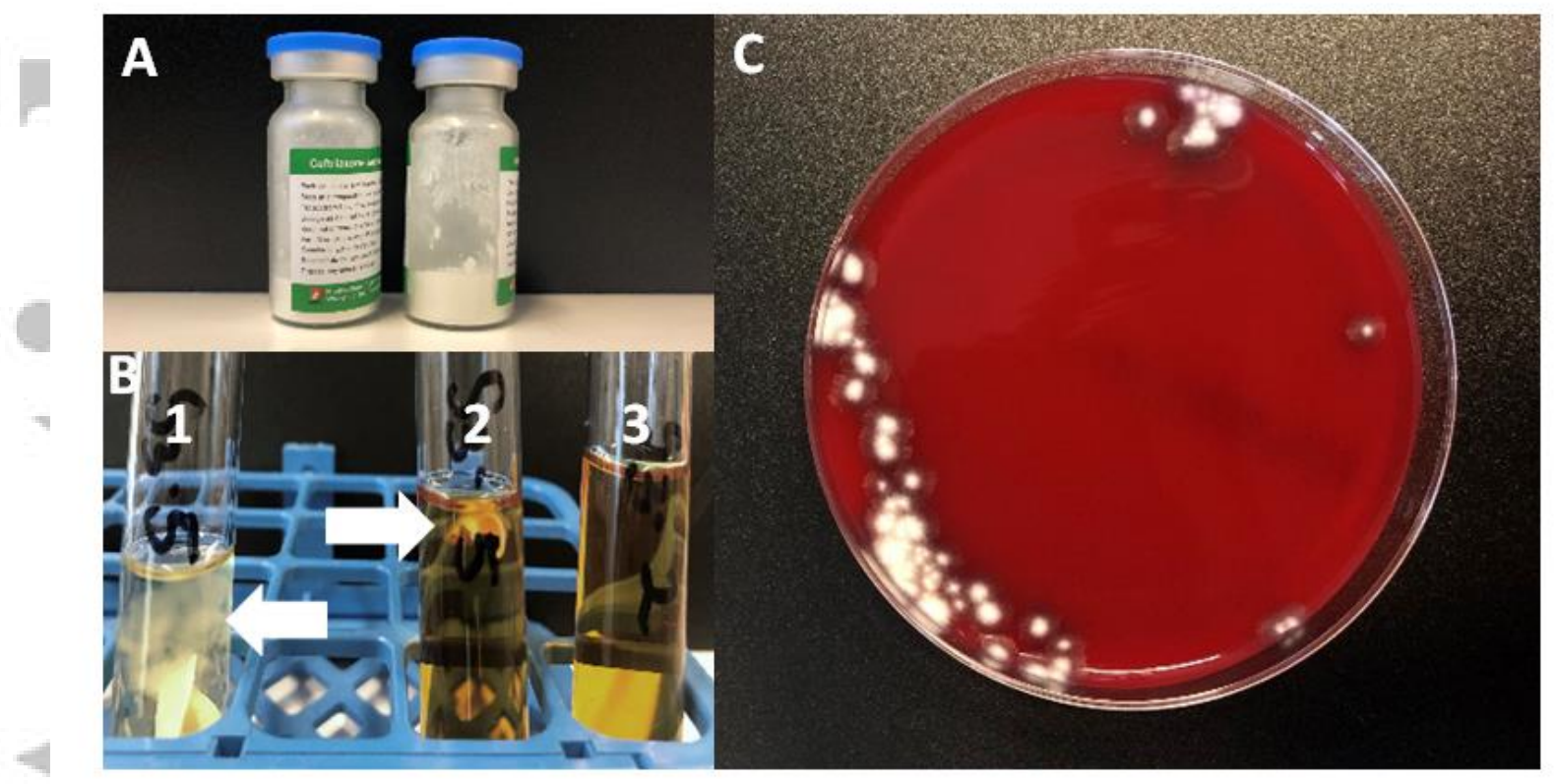

Figure 1: Sterility testing of SF ceftriaxone in TSB broth.

A: SF injectable sample of ceftriaxone; B (1): test for sterility after membrane filtration, (2) test for sterility by direct inoculation, (3) negative control. C: Isolated fungal strain, originating from the sterility test, plated on Columbia $+5 \%$ Sheep Blood agar and incubated at $37^{\circ} \mathrm{C}$ for 3 days.

This article is protected by copyright. All rights reserved. 
A

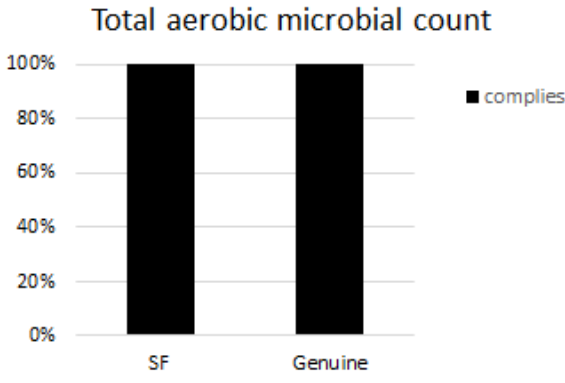

C 500

45

400

긴 $^{350}$

过 300

这 250

亭 200

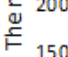

B

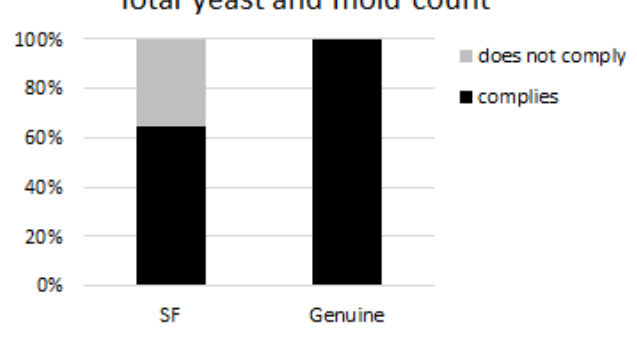

100

50

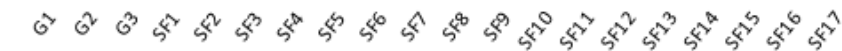

Figure 2: Outcome of the bioburden testing on the different SF antimicrobials.

A: Histograms representing all samples that comply with total aerobic microbial count (TAMC); B: Histograms representing the percent of compliant or noncompliant samples for total yeast and mold count (TYMC); C: The number of colony forming units (CFU) of TYMC for each sample. The pharmacopoeial limit (CFU=20) is indicated by the dotted line. * indicates that the counting results are not $100 \%$ accurate due to the pronounced growth of filamentous fungi.

This article is protected by copyright. All rights reserved. 


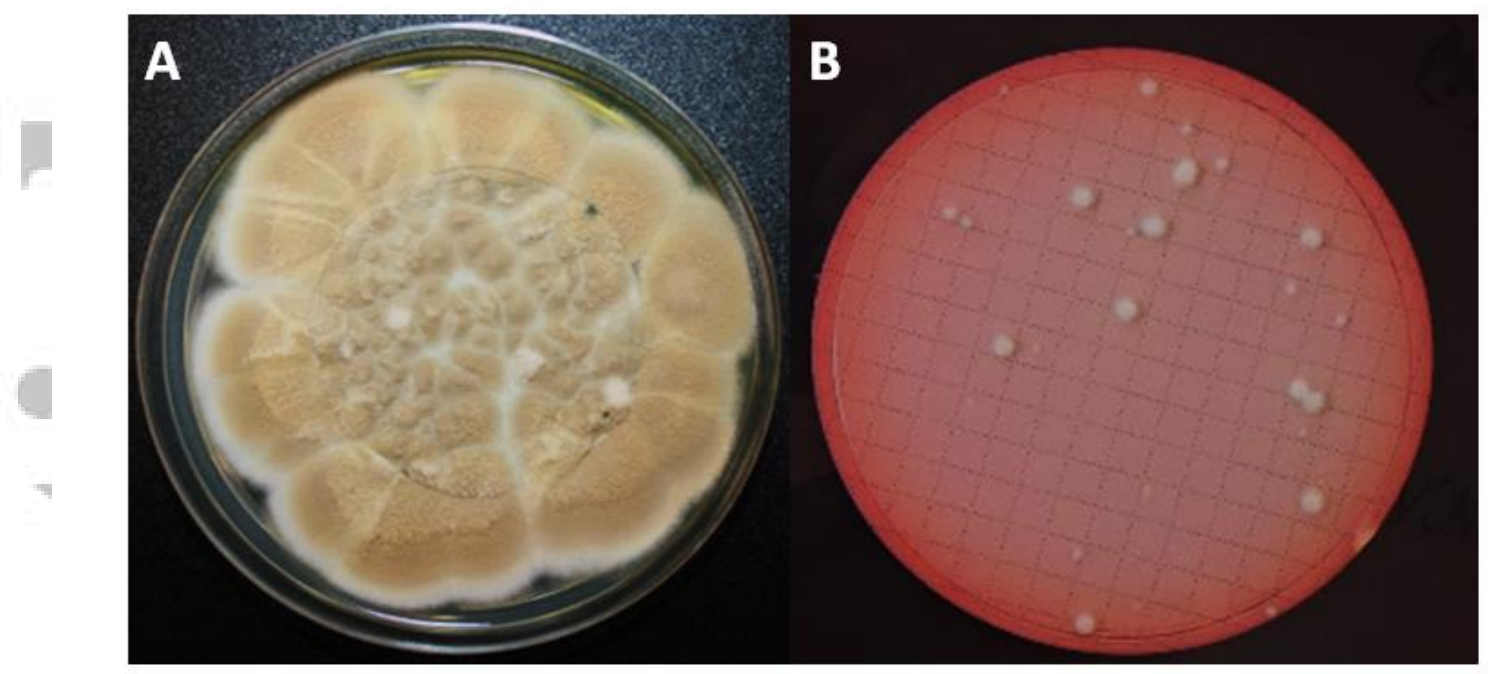

Figure 3: Examples of heavy contaminated samples.

A: amoxicillin sample (SF 5) tested on SCG plate; B: tetracycline sample (SF15) tested on MCV plate.

This article is protected by copyright. All rights reserved. 